\title{
Management of Prescription Refills in Primary Care: An Oklahoma Physicians Resource/Research Network (OKPRN) Study
}

\author{
Charles W. Ferrell, Cheryl B. Aspy, PhD, and James W. Mold, MD, MPH
}

Background: Management of prescription refills is a significant challenge for primary care practices, but little information exists on the best way to do it.

Methods: Using the "best practices research" method within a primary care practice-based research network, we delineated steps involved in the process and the most important requirements for each step. We identified potential exemplary practices for each step and conducted practice audits and patient surveys to document actual performance. Methods with the highest performance ratings for the predetermined requirements for the steps were combined into a "best" unified approach.

Results: Half of the surveyed practices were satisfied with at least one step in their current refill management process, but only $9 \%$ (3 practices) were satisfied with all 5 steps. Practice audits identified acceptable methods for each step. The best combined method involves teaching patients to call the pharmacy first for prescription refills, accepting only fax requests from the pharmacy, allowing a nurse or medical assistant to make most refill decisions, documenting them in the chart immediately, responding back to the pharmacy by fax, and relying on the pharmacy to notify the patient when the refill is ready. Patient satisfaction with this method was reasonably good. The cost per refill was approximately $\$ 0.25$ excluding overhead associated with office equipment and utilities.

Conclusions: A satisfactory method for managing prescription refills in primary care practices was identified using the best practices research method. (J Am Board Fam Med 2006;19:31-8.)

Primary care physicians rely heavily on the use of medications for treating patients. It has been estimated that $42 \%$ to $71 \%$ of visits to a physician result in at least one prescription for medication. ${ }^{1,2}$ One study demonstrated that of all telephone calls made to a primary care physician's office, $31 \%$ resulted in a prescription, and of these, $44.9 \%$ involved the refill of a previously written prescription. ${ }^{3}$ Primary care practices spend a significant amount of time and resources in the management of prescription refills, and it is, therefore, very important that they have an accurate and efficient system in place for doing so.

Very few studies have addressed methods for managing prescription refills. Those that have were

Submitted 8 April 2005; revised 9 September 2005; accepted 13 September 2005 .

From the University of Oklahoma College of Medicine (CWF); and Department of Family and Preventive Medicine, University of Oklahoma Health Sciences Center, Oklahoma City, OK (CBA, JWM)

Conflict of interest: none declared.

Corresponding author: Cheryl B. Aspy, PhD, Department of Family and Preventive Medicine, University of Oklahoma Health Sciences Center, 900 NE 10th Street, Oklahoma City, OK 73104 (E-mail: Cheryl-aspy@ouhsc.edu). conducted in unique settings or have addressed narrow aspects of the problem. For example, a recent study explored giving pharmacists the authority to refill chronic medications for patients in a Veterans Affairs hospital system until a visit could be scheduled with the patient's physician. ${ }^{4}$ Other studies have examined ways to improve documentation of prescriptions in the medical record, ${ }^{5}$ the efficiency of a medical screening clinic that employed a pharmacist as a part of the health care team, ${ }^{6}$ and the use of E-mail for refill requests. ${ }^{7}$

The purpose of this study was to identify and describe at least one effective and efficient method for managing prescription refills in primary care practice settings. We used a research method that we have called "best practices research." management of prescription refills is a common challenge faced by virtually all primary care physicians, we believed that we could find clinicians who had already developed components of an effective and efficient refill management system. Best practices research attempts to tap into the wisdom of practicing clinicians to identify effective, fieldtested solutions or partial solutions, combining the best of these into a single unified approach. We 
have used this method to identify effective ways to increase pneumococcal immunization rates, ${ }^{9}$ manage laboratory test results, ${ }^{10}$ and improve the quality of care provided to patients with diabetes. ${ }^{11}$

\section{Methods}

Best practices research involves: (1) identification of a series of steps involved in the process being investigated, (2) agreement on a definition of best for each step, (3) identification and evaluation of promising approaches to individual steps, (4) combination of best methods for each step into a unified method, and (5) evaluation of the combined method. ${ }^{8}$ In this case, the following specific approaches were used.

\section{Identification of Steps Involved in the Process}

During a semiannual convocation, physician members of the Oklahoma Physicians Resource/Research Network (OKPRN) identified the steps involved in the management of prescription refills. Subsequently, office staff who were attending an OKPRN staff training conference were asked to reflect on these steps and revise them. The revised set of steps was then critiqued by a group of pharmacists. This revision was sent to all members of OKPRN over its listserv for review and comment. Through this process, 5 steps were agreed upon, not necessarily occurring in this order: (1) patient access to the process, (2) communication between clinic and pharmacy, (3) decision-making by clinic staff, (4) patient notification, and (5) medical record documentation.

Reduction of the frequency of refill requests by providing more doses and more refills per prescription was also discussed. Some physicians reported that they limit the number of refills given to a patient to ensure follow-up. Others suggested writing for the maximum number of refills allowed and creating a separate system for tracking adherence to follow-up appointments. We chose not to evaluate this aspect of the process in this study while acknowledging its potential importance.

\section{Definition of Best}

Following the identification of steps, a committee consisting of the authors [a second year medical student (CWF), an academic family physician (JWM), a PhD faculty member (CBA)], met to define the meaning of best for each step. A list of possible requirements for each step was generated through brainstorming; after which, each was discussed and consensus was reached regarding the top 2 requirements and their order of importance.

For all 5 steps, the group agreed that a good prescription refill management process required accuracy, efficiency, acceptability for patients and office staff, and applicability to a wide range of primary care practices. However, these requirements were prioritized differently for each step. The top 2, prioritized requirements for step 1, were efficiency and patient satisfaction, in that order; for step 2, accuracy and efficiency; for step 3, accuracy and efficiency; for step 4, patient satisfaction and efficiency; and for step 5, accuracy and efficiency.

Generalizability and lack of interference with other office activities were considered important for all steps but were not considered independently for each step and were not specifically evaluated in the practice audits.

\section{Identification and Evaluation of Potential Best Methods for Individual Steps}

Surveys were faxed to 65 physicians in the 49 OKPRN member practices. On separate copies of this survey, the physician, a member of the nursing staff, and a key office staff member were asked to independently rate their level of satisfaction with their office's management of each of the 5 steps of the prescription refill process on a 5 -point Likert scale with 1 being not satisfied and 5 being completely satisfied. These surveys were returned by fax to the University of Oklahoma Health Sciences Center (OUHSC) where they were evaluated. Physician offices at which a physician did not respond $(\mathrm{N}=3)$ or where only the physician responded $(\mathrm{N}=7)$ were excluded from further evaluation. Physician/staff teams that showed high satisfaction rates (mean rating $\geq 4$ ) for at least one step of their prescription management system were queried further about those component(s) of their process with which they were satisfied $(\mathrm{N}=17)$. Five practices were selected for in-depth audits; practices were purposefully chosen from those satisfied with at least one step in the process in such a way as to insure that the full spectrum of different methods for each step was represented in the sample. At least 2 potentially exemplary practices were chosen for each step. 


\section{Measures}

Outcome measures related to patient satisfaction (steps 1 and 4) were obtained from a mailed survey. This survey was sent to each patient who had requested a refill during the 1-week audited period in each practice. Patients were asked how they notified the physician's office regarding refills, how long the refill process took, how satisfied they were with the way they were notified when the prescription was ready, and how satisfied they were with the process as a whole (each on a 5-point Likert scale).

For step 2, communication with the pharmacy, an error was defined as any event that delayed or distorted communication. These errors were often associated with faxing errors, eg, a fax did not go through, multiple faxes were sent, or an incorrect fax number was used. Errors related to decisionmaking (step 3) were defined as decisions made by the medical assistant or LPN that were different from those the physician would have made. These were determined by examining all refill decisions made during 1 week, comparing the decision made to the decision the physicians said they would have made for each request. Decisions were made by the physician in 2 clinics, and therefore, no errors were attributed to these clinics for this step. A documentation error (step 5) resulted when the refill was not documented in the chart. Medical records were reviewed to determine whether proper documentation had occurred (step 5). Error rates were calculated using the number of prescription refill requests as the denominator for each of the 5 sites.

Efficiency was operationalized as the cost to the practice. Cost was computed by determining the time involved for each employee to complete the tasks associated with each step and multiplying it by the average hourly salaries of the employees involved. A medical student (CWF) timed each component of the process from request initiation through decision-making and contact with the pharmacy for at least 25 patients at each site. Cost calculations were bundled into communication (including receiving and returning faxes and pulling charts) and decision-making.

\section{Analysis}

Data from the audits and from the patient satisfaction responses were entered into an Excel spreadsheet and uploaded into SimStat for analyses. Descriptive statistics were computed for all variables and comparisons across practices for categorical data were made using $\chi^{2}$ analyses of cross-tabulated data. For continuous variables, comparisons across practices were made using analysis of variance (ANOVA). For all analyses, the significance level was set at 0.05 . For the patient surveys, a power analysis suggested that we would be able to detect a $5 \%$ or greater difference in proportions $80 \%$ of the time with a sample size of 24 so we decided to survey all patients requesting refills in a given week ( $\mathrm{N}=31$ to 91 in the audited practices). Field notes were kept by the practice auditor (CWF), and these were reviewed with the other authors to identify important themes. This study was reviewed and approved by the university's Institutional Review Board.

\section{Combining Best Methods}

Based on the performance of the methods used in the audited practices for the individual steps, in accordance with our predetermined requirements and criteria, best approaches were identified for each step and then combined into a single best method for managing prescription refills. The cost of the best combined method was estimated by averaging the cost for practices using the best method for each step and then summing those averages.

\section{Results}

Responses to the initial survey were received from 36 of the 49 practices (73\%), 41 of 65 physicians $(63 \%), 34$ of the 65 nursing staff $(52 \%)$, and 29 of the 65 office staff (45\%). However, 7 of the physician responses were not accompanied by either a nurse or staff member survey resulting in an eligible sample of 34 physician/staff teams for a response rate of $52 \%$. Mean satisfaction scores were greater than or equal to 4 in $26 \%$ of the practices for step 1, in $21 \%$ for steps 2 and 4 , in $32 \%$ for step 3 , and in $35 \%$ for step 5 . Of the sample ( $\mathrm{N}=17)$, $50 \%$ were not satisfied with any step in their process. Five of the teams (15\%) were satisfied with only 1 step in their process, 4 teams $(12 \%)$ with 2 steps, 2 teams (5\%) with 3 steps, and 3 teams (9\%) each were satisfied with 4 and 5 steps of their process. The average number of prescription refill requests was 63 per week (range 31 to 91) per physician in the 5 audited practices. A description of the practices that participated in this study can be found in Table 1 . 


\section{Step 1: Patient Access to the System}

Efficiency and patient satisfaction were the primary requirements for this step. Efficiency for the practice was dramatically improved by having the pharmacy handle initial patient phone calls. Not only did the practice save time on phone calls back and forth to patients, the information received from the pharmacy was more complete and accurate than that obtained from patients. Cost information for this step is included in the cost of clinical decisionmaking (Table 4).

For patient satisfaction, data from patient surveys were used to determine the best method. The mean response rate to the patient surveys was $46 \%$ (range $41 \%$ to $54 \%$ ). Results related to this step can be found in Table 2. Four of the 5 audited clinics (clinics A, B, C, and E) said that they require patients to call the pharmacy for prescription refills. In these clinics, $55 \%$ to $88 \%$ of patients actually used this method. The other clinic allowed patients to call the office but recommended that they call the pharmacy first. In this practice (D), only $33 \%$ of survey respondents called the pharmacy first. No patients reported using E-mail to request prescription refills.

Patients seemed to prefer calling the practice rather than the pharmacy for prescription refills. However, even in clinic E, which had the lowest patient satisfaction rate, patients on average were more than satisfied with the method. The majority of patients (56\%) reported that it took less than 2 minutes to make the initial contact with the system and the differences among sites were not signifi- cant. The time required for patient access was not related to whether they called the pharmacy or the physician's office.

\section{Step 2: Communication with Pharmacies}

The critical requirements for communicating with the pharmacy were accuracy and efficiency. In the practices satisfied with their management of this step (clinics C, D, and A), pharmacies communicated with the practice by fax, and decisions of the practice were faxed back to the pharmacy. In clinic $\mathrm{E}$, a voice mail option is available and requests come directly to the medical assistant charged with making the decision. In clinics $\mathrm{B}$ and $\mathrm{E}$, an electronic record system makes location and retrieval of the chart more efficient.

Errors associated with these methods differed significantly across the sites (see Table 3). Clinics A and $\mathrm{E}$ both had more errors than would be expected by chance compared with the other.

Costs per refill request related to communication with the pharmacy as well as mean practice satisfaction scores for clinics A through E can also be found in Table 3 . These costs include the time required to pull and refile charts. Clinic B had the lowest cost per interaction (\$0.06) for this step primarily because of an efficient electronic medical record (EMR). For all other clinics, the cost was more than twice as high because of the time and effort required to pull and refile the record. Clinic $\mathrm{E}$ had the highest cost $(\$ 0.33)$ per transaction despite the use of an electronic record. This seemed to be because of the multiplicity of methods used

Table 1. Characteristics of the Five Audited Practices

\begin{tabular}{|c|c|c|c|c|c|}
\hline \multirow[b]{2}{*}{ Characteristic } & \multicolumn{5}{|c|}{ Physician } \\
\hline & A & B & $\mathrm{C}$ & $\mathrm{D}$ & $\mathrm{E}$ \\
\hline Practice type & Small group* & Solo & Small group* & Solo & Residency faculty \\
\hline $\begin{array}{l}\text { Average number of patients } \\
\text { seen per week }\end{array}$ & 125 & 108 & 75 & 70 & 40 \\
\hline $\begin{array}{l}\text { Payor mix: Medicare/Medicaid } \\
\text { Commercial insurance } \\
\text { Self-pay }\end{array}$ & $\begin{array}{l}30 \% \\
59 \% \\
11 \%\end{array}$ & Not available & $\begin{array}{l}65 \% \\
35 \% \text { combined } \\
0 \%\end{array}$ & $\begin{array}{l}25 \% \\
50 \% \\
25 \%\end{array}$ & Not available \\
\hline Scope of practice & No OB & No OB & No OB procedures & No OB & Full scope \\
\hline Has an EMR & No & Yes & No & No & Yes \\
\hline Geographic location & Suburbs & Rural & Rural & Rural & Urban \\
\hline Size of town & $>50,000-<100,000$ & $<5,000$ & $>5,000-<30,000$ & $<5,000$ & $>250,000$ \\
\hline Pharmacies available & National chains & $>2$ locally owned & National chains & $>2$ locally owned & National chains \\
\hline $\begin{array}{l}\text { Steps for which practice } \\
\text { satisfaction was high }\end{array}$ & $3,4,5$ & 1,5 & 2 & $1,2,3,4,5$ & 1,3 \\
\hline
\end{tabular}

*Less than 6 clinicians. 
Table 2. Step 1: Patient Access to the System

\begin{tabular}{lcccc}
\hline Site* & $\begin{array}{c}\text { Percentage Calling } \\
\text { Pharmacy First }\end{array}$ & $\begin{array}{c}\text { Mean (SD) Patient } \\
\text { Satisfaction with Method } \neq\end{array}$ & $\begin{array}{c}\text { Percentage Reporting }<2 \\
\text { Minutes for Initial Contact\$ }\end{array}$ & $\begin{array}{c}\text { Mean Practice } \\
\text { Satisfaction }\end{array}$ \\
\hline Clinic D\| & 33 & $4.3(0.99)$ & 60 & 4.0 \\
Clinic B & 55 & $4.0(1.3)$ & 56 & 4.7 \\
Clinic A & 86 & $3.9(0.95)$ & 42 & 2.3 \\
Clinic C & 77 & $3.5(1.1)$ & 67 & 3.0 \\
Clinic E & 88 & $3.2(1.2)$ & 50 & 4.0 \\
\hline
\end{tabular}

* Table is ordered high to low based on patient satisfaction.

$\dagger \chi^{2}=20.1, \mathrm{df}=4, P<.0001$.

$\ddagger \mathrm{F}_{4,128}=3.5, P=.011 ;$ Newman-Keuls post hoc test revealed significant differences between clinics $\mathrm{A}$ and $\mathrm{E}(P=.03) ; \mathrm{B}$ and $\mathrm{E}(P=$ $.03)$; and $\mathrm{D}$ and $\mathrm{E}(P=.02)$.

$\S \chi^{2}=4.2, \mathrm{df}=4, P=.37$.

\| Self-reported practice satisfaction rates of 4.0 or higher.

for communicating with both patients and pharmacies. In addition, the electronic record system used in this practice had a relatively inefficient method for documenting medication refills.

Comparing clinic B, which uses an efficient EMR to the average cost of all other clinics $(\$ 0.20)$, and assuming an average of 63 prescription refill requests per week per clinician, an EMR seemed to save the practice approximately $\$ 459$ per clinician per year for this function alone $(\$ 0.14 \times 63 \times 52=$ \$458.64).

\section{Step 3: Decision-making by Clinic Staff}

The primary requirements for this step were also accuracy and efficiency. Table 4 contains the qualifications of the principal decision-maker, whether he/she uses a written protocol, the error rates, and the cost per refill for this step. A $\chi^{2}$ analysis found significant differences in error rates for the clinics. Clinics B and E both had significantly higher error rates than would be expected by chance alone. Clinic E had the highest error rate for this step among the audited practices despite their use of an EMR and a written protocol. The auditor observed that the errors were virtually all errors of omission. That is, the decision-maker refused refills that the physician would have approved. The written protocols were too rigid to accommodate the wide variety of patient circumstances encountered.

Costs varied widely for this step. Much of this had to do with the cost of the decision-maker (physicians' time costs more than medical assistants and licensed practical nurses time). However, some persons were simply more efficient than others were as well. Clinic A had both the lowest cost and the lowest error rate for this step. Although clinics $\mathrm{C}$ and $\mathrm{D}$ also had a $0 \%$ error rate, their average costs per request were greater (2 and 4 times, respectively) than clinic A. The medical assistant making the refill decisions at clinic A had worked in that practice with the same physician for a number of years and was very efficient. Although satisfied with their methods, clinics D and E had the highest costs.

Table 3. Step 2: Communication with Pharmacies

\begin{tabular}{llccc}
\hline Site* & Method Used & $\begin{array}{c}\text { Error Rate } \\
(\%) \dagger\end{array}$ & $\begin{array}{c}\text { Mean Cost Per Refill for } \\
\text { the Communication Step }\end{array}$ & $\begin{array}{c}\text { Mean Practice } \\
\text { Satisfaction Level }\end{array}$ \\
\hline Clinic D\| & FAX & 0 & $\$ 0.18$ & 4.0 \\
Clinic B\$ & FAX/voice mail & 2 & $\$ 0.06$ & 3.7 \\
Clinic C\| & FAX & 2 & $\$ 0.13$ & 4.0 \\
Clinic A\$ & FAX/phone & 8 & $\$ 0.17$ & 2.3 \\
Clinic E & FAX/phone & 10 & $\$ 0.33$ & 3.3 \\
\hline
\end{tabular}

*Table is ordered low to high based on error rate.

$\dagger \chi^{2}=12.6, \mathrm{df}=4, P=.013$.

$\ddagger$ Includes chart retrieval.

$\$$ Self-reported practice satisfaction rates of 4.0 or higher. 
Table 4. Step 3: Decision-Making

\begin{tabular}{lcccccc}
\hline Site* & Decision-Maker & $\begin{array}{c}\text { Written } \\
\text { Protocol }\end{array}$ & EMR† & $\begin{array}{c}\text { Error Rate } \\
(\%)^{\ddagger}\end{array}$ & $\begin{array}{c}\text { Mean Cost for } \\
\text { Refill Decision }\end{array}$ & $\begin{array}{c}\text { Mean Practice } \\
\text { Satisfaction Level }\end{array}$ \\
\hline Clinic A\$ & MA & No & No & 0 & $\$ 0.10$ & 4.7 \\
Clinic C & MD & No & No & 0 & $\$ 0.21$ & 2.5 \\
Clinic D\$ & MD & No & No & 0 & $\$ 0.82$ & 5.0 \\
Clinic B & LPN & Yes & Yes & 4 & $\$ 0.11$ & 3.7 \\
Clinic ES & MA & Yes & Yes & 10 & $\$ 0.36$ & 4.0 \\
\hline
\end{tabular}

* Table is ordered low to high based on error rate.

† EMR, electronic medical record; MA, medical assistant; LPN, licensed practical nurse.

$\ddagger \chi^{2}=19.6, \mathrm{df}=4, P=.001$.

$\$$ Self-reported practice satisfaction rates of 4.0 or higher.

\section{Step 4: Notification of Patients}

The critical requirements for this step were efficiency and patient satisfaction. All 5 clinics leave this step to the pharmacies. Patients are routinely informed that they are to contact the pharmacy before the physician's office closes so that if an error has occurred between the pharmacy and the physician's office, it can be remedied before the next day. If it is time for the patient to schedule an appointment with the physician, they are typically granted a 1-month supply and it is noted on the faxed request or the pharmacist is informed on the phone that the patient needs to schedule an appointment and that they will receive no more refills until that happens. If the refill is denied, as is often the case with narcotics, a reason is noted on the fax message to the pharmacy. However, with a denial, the physician's office usually calls the patient to explain why the refill was denied. As for step 1, it is much more efficient for the practice to rely on the pharmacy to contact patients when the prescription is ready.

When asked about the total time required to get a prescription refilled, the majority of patients reported the total time as less than 8 hours (see Table 5). However, there was a significant difference among the practices with clinic D having the highest proportion with total refill times less than 8 hours and clinic $\mathrm{E}$ having the lowest. There was no significant difference among the 5 sites for patient satisfaction with their notification methods when means were compared. Patient satisfaction was lowest for clinic E and highest for clinic D. Mean practice satisfaction generally followed the same ranking as the proportion of patients reporting total prescription refill time as less than 8 hours. For example, clinic D had the highest mean practice satisfaction level and also the highest percentage of patients reporting less than 8 total hours for the refill process.

\section{Step 5: Documentation}

Accuracy was the primary requirement for the documentation process. In clinic A, a copy of the fax is attached to the chart, and then sent to the transcriptionist who transcribes the refill into the record. Clinics B and D use a similar method and had even lower error rates. Error rates and mean practice satisfaction scores can be found in Table 6 .

The less satisfied practices used very different methods. In clinic $\mathrm{C}$, the decision-maker documents the refill on a handwritten $\log$ in the patient's record at the time of the decision with an error rate of $2 \%$. In clinic $\mathrm{E}$, where the decisionmaker documents the refill in the electronic medical record at the time of the decision, no errors were found. These differences in error rates were not statistically significant $\left(\chi^{2}=3.3, \mathrm{df}=4, P>\right.$

Table 5. Step 4: Notification of Patients

\begin{tabular}{lccc}
\hline & $\begin{array}{c}\text { Percentage of Patients } \\
\text { Reporting Less } \\
\text { Than 8 Hours for } \\
\text { Total Refill Time† }\end{array}$ & $\begin{array}{c}\text { Mean } \\
\text { Patisfaction }\end{array}$ & $\begin{array}{c}\text { Practice } \\
\text { Satisfaction } \\
\text { Level }\end{array}$ \\
\hline Clinic D\$ & 88 & 3.9 & 5.0 \\
Clinic B & 70 & 3.9 & 3.3 \\
Clinic A\$ & 76 & 3.6 & 4.0 \\
Clinic C & 61 & 3.6 & 3.0 \\
Clinic E & 31 & 3.0 & 3.3 \\
\hline
\end{tabular}

*Table is ordered high to low based on patient satisfaction. $\dagger \chi^{2}=17.6, \mathrm{df}=4, P<.01$.

$\ddagger$ Nonsignificant: $\mathrm{F}_{4,124}=2.2, P=.07$.

$\S$ Self-reported practice satisfaction rates of 4.0 or higher. 
Table 6. Step 5: Documentation

\begin{tabular}{lccc}
\hline Site* & EMR† & $\begin{array}{c}\text { Error Rate } \\
(\%) \neq\end{array}$ & $\begin{array}{c}\text { Mean Practice } \\
\text { Satisfaction Level }\end{array}$ \\
\hline Clinic E & Yes & 0 & 3.7 \\
Clinic D $\$$ & No & 0 & 5.0 \\
Clinic B $\$$ & Yes & 1 & 4.3 \\
Clinic C & No & 2 & 3.0 \\
Clinic A $\$$ & No & 3 & 4.0 \\
\hline
\end{tabular}

*Table is ordered low to high based on error rate.

$\dagger \mathrm{EMR}$, electronic medical record.

$\ddagger \chi^{2}=3.3, \mathrm{df}=4, P>.05$.

$\$$ Self-reported practice satisfaction rates of 4.0 or higher.

$.05)$. The best methods for each step of the prescription refill process can be found in Table 7 .

\section{Discussion}

This is the first attempt that we are aware of to identify an effective, comprehensive system for the management of prescription refills in primary care settings. Although much more work needs to be done, we learned some important principles and can tentatively recommend an approach to this task. This is an important contribution because most practices that we surveyed were not satisfied with the way they currently manage prescription refills. Given the many times they must perform the task each day, it is evident that a problem exists that should be addressed.

Some of the principles learned during this study resemble those learned previously. ${ }^{10,11}$ Single option methods (eg, asking all patients to call the pharmacy first) tend to work better than multiple option methods. Time spent by staff on the telephone is expensive, and physician time is particularly expensive and not always needed for routine tasks. Time spent pulling, transporting, and refiling paper charts is also costly; $\$ 459$ per clinician per year just for the management of prescription refills. Error rates are generally higher than physicians think they are.

The best method identified for steps 1 (patient access) and 2 (communication with the pharmacy) is to require that the patient contact the pharmacy first and have the pharmacy fax requests to the physician's office. The pharmacy should also be responsible for notifying the patient that the prescription has been approved and is ready to be picked up (step 4, notification of patient). This shifts overhead from physicians' offices, which are not reimbursed for refill services, to the pharmacies, which profit from the sale of the medication. Because faxed requests are computer generated, they are legible and therefore likely to reduce errors compared with patient or pharmacy phone calls to the practice in which necessary information such as correct dosage or amount requested are often omitted. It does not, however, reduce the time that it takes for patients to contact the system, and it results in some reduction in patient satisfaction. Decisions regarding the use of this method should be tempered by this reality; however, a majority of patients reported being very or completely satisfied with their physician's method for managing refills. Patients also reported high levels of satisfaction with relying on the pharmacy to let them know when a prescription had been refilled.

Based on our data, medical assistants and licensed practical nurses are capable of making accurate decisions about the vast majority of medication refill requests (step 3). Obviously some training is required, and the default should always be to the physician in questionable cases. An interesting finding was that offices with a written protocol for their decision-makers to follow (clinics B and E) had higher rates of decision-making errors than those who had only verbal instructions. This

Table 7. Best Method for Each Step

\begin{tabular}{ll}
\hline Steps in the Prescription Refill Process & Best Method \\
\hline $\begin{array}{l}\text { Step 1. Patient access to the system } \\
\text { Step 2. Communication with the pharmacy }\end{array}$ & $\begin{array}{l}\text { Patients should contact the pharmacy first when a refill is needed. } \\
\text { Communication should be by FAX and initiated by the pharmacy. } \\
\text { Least costly method is to have nurse or medical assistant make decisions regarding } \\
\text { refills. Training with the physician, including feedback regarding decisions will } \\
\text { improve accuracy. Written protocols were associated with increased error rates. }\end{array}$ \\
$\begin{array}{l}\text { Step 4. Notification of the patient } \\
\text { Ttep 5. Documentation }\end{array}$ & $\begin{array}{c}\text { Thenarmacy should notify the patient regarding the prescription refill decision. } \\
\text { make this more efficient. }\end{array}$ \\
\hline
\end{tabular}


might have been because of the varied experience levels of the staff in the 5 clinics or it may be a chance finding. However, it is more likely that, because of the wide variety of patients seen in primary care settings, there are many legitimate exceptions to well-intended rules.

Documentation (step 5) should be done by the person making the refill decision. This seems to reduce the rate of errors made during transfer of paperwork. For EMRs, easy and accurate documentation and efficient access to the patient's prescription list are essential features not always present in existing programs. Some of the audited practices designated certain times of each day for handling prescription refills. Others handled them as they came in as time permitted. A summary of the best practice steps can be found in Table 7 .

The study has several limitations. Audits were conducted only in practices that reported high satisfaction with their methods for at least one of the management steps. Satisfaction may or may not be an indicator of the effectiveness of the method or of an exemplary practice. That there were more similarities among their methods than differences adds support to the validity of our method for identifying exemplars and to our conclusions and recommendations. However, it is possible that better methods existed in practices that failed to respond or had higher thresholds for reporting satisfaction. Only 5 practices were audited, and we did not identify and audit practices with very low satisfaction rates as a basis for comparison. Response rates to the patient satisfaction survey were only approximately $50 \%$. We also have not yet tested the combined best practice method against alternatives. None of the practices were using E-mail to communicate with pharmacies or with patients.

However, we believe that the consistency of methods used by the satisfied practices speaks to the veracity of our results and recommendations. In addition, when we have discussed our findings at both network and national meetings, attendees report that they have significant face validity. All the steps have been field tested in active clinical practices and seem to be feasible and acceptable. The combined best method is broadly generalizable. Whereas an electronic medical record can make the process more efficient depending to some degree on the software, the method is equally effective using paper records.

We are grateful for the contributions of the office and nursing staffs and physicians of the OKPRN who participated in this study.

\section{References}

1. Collyer JA. A measure of a family doctor's work. Part II: drugs, time, charges, morbidity. CMA 1975;112: 135-8.

2. Bain DJ, Haines AJ. A year's study of drug prescribing in general practice using computer assisted records. J R Coll Gen Pract 1975;25:41-8.

3. Spencer DC, Daugird AJ. The nature and content of telephone prescribing habits in a community practice. Fam Med 1990;22:205-9.

4. Hasson N, Carmichael J. VA hospital program targets zero-refill problem. Clin Resour Manag 2000; $1: 120-2$.

5. Miller LG, Matson CC, Rogers JC. Improving prescription documentation in the ambulatory setting. Fam Pract Res J 1992;12:421-9.

6. Morton WA, Bridges ME. Pharmaceutical services in a medical screening clinic. Am J Hosp Pharm 1978;35:574-8.

7. Couchman GR, Forjuoh SN, Rascoe TG. E-mail communications in family practice: what do patients expect?. J Fam Pract 2001;50:414-8.

8. Mold JW, Gregory M. Best practices research. Fam Med. 2003;35:131-4.

9. Reilly K. Use of standing orders to improve compliance with vaccination recommendations. Annual Meeting of the North American Care Research Group 1996; Vancouver, British Columbia, Canada.

10. Mold JW, Cacy DS, Dalbir DK. Management of laboratory test results in family practice. J Fam Pract 2000;49:709-15.

11. Nagykaldi Z, Mold JW, Diabetes patient tracker, a personal digital assistant-based diabetes management system for primary care practices in Oklahoma. Diabetes Technol Ther 2003;5:997-1001. 